\title{
L'ordre des choses : cabinets et musées d'histoire naturelle au Québec (1824-1900)
}

\section{Raymond Duchesne et Paul Carle}

Volume 44, numéro 1, été 1990

URI : https://id.erudit.org/iderudit/304861ar

DOI : https://doi.org/10.7202/304861ar

Aller au sommaire du numéro

Éditeur(s)

Institut d'histoire de l'Amérique française

ISSN

0035-2357 (imprimé)

1492-1383 (numérique)

Découvrir la revue

Citer cet article

Duchesne, R. \& Carle, P. (1990). L'ordre des choses : cabinets et musées d'histoire naturelle au Québec (1824-1900). Revue d'histoire de l'Amérique française, 44(1), 3-30. https://doi.org/10.7202/304861ar
Résumé de l'article

$\mathrm{Au}$ XIX ${ }^{\mathrm{e}}$ siècle, la multiplication des musées et des cabinets d'histoire naturelle au Québec est liée à la popularité de cette science dans les classes moyennes et au développement de l'enseignement scientifique dans les collèges et les universités. Notre étude indique que ce phénomène relève davantage de la mode et de l'histoire des mentalités que de la professionnalisation des sciences. Sous ce rapport, l'histoire des musées du Québec ne se distingue guère de celle des musées étrangers. 


\title{
L'ORDRE DES CHOSES: CABINETS ET MUSÉES D'HISTOIRE NATURELLE AU QUÉBEC (1824-1900) ${ }^{1}$
}

\author{
RAYMOND DUCHESNE \\ Télé-Université \\ Université du Québec
}

PAUL CARLE

CREST - Département des communications Université du Québec à Montréal

\begin{abstract}
RÉSUMÉ
Au XIXe siècle, la multiplication des musées et des cabinets d'histoire naturelle au Québec est liée à la popularité de cette science dans les classes moyennes et au développement de l'enseignement scientifique dans les collèges et les universités. Notre étude indique que ce phénomène relève davantage de la mode et de l'histoire des mentalités que de la professionnalisation des sciences. Sous ce rapport, l'histoire des musées du Québec ne se distingue guère de celle des musées étrangers.
\end{abstract}

\begin{abstract}
Throughout the nineteenth century, natural history museums proliferated in Quebec. Of course, the popularity of such museums was a consequence of the popularity of natural history itself, as a form of diversion among the middle-class. It was linked also to the teaching of the various branches of the discipline at the colleges and universities of the province. Our study shows that the popularity of natural history had more to do with social and cultural trends than with the professionalization of science. In this respect, the "museum movement" in Quebec did not separate itself from what was observed in the rest of the world at the time.
\end{abstract}

La période qui s'étend de 1870 à 1900 est considérée comme l'«âge d'or» des musées d'histoire naturelle ${ }^{2}$. C'est à cette époque qu'atteignent leur apogée les grandes institutions nationales comme le Muséum d'histoire naturelle de Paris, le Natural History Museum de Londres, partie du British Museum, ou le U. S. Natural History Museum de

1 Les auteurs désirent exprimer leur reconnaissance au Conseil de recherches en sciences humaines du Canada, au Fonds FCAR et à la Télé-Université pour l'appui obtenu au cours de leurs recherches.

2 Edward P. Alexander, Museums in Motion. An Introduction to the History and Function of Museums (Nashville, 1979), 50-60; Kenneth Hudson, Museums of Influence (Cambridge, 1987), 65ss; Susan Sheets-Pyenson, Cathedrals of Science: the Development of Colonial Natural History Museums During the Late Nineteenth-Century (Montréal, 1988), 5-12. 
Washington $^{3}$. Cet «âge d'or» de la muséologie scientifique est l'aboutissement d'un mouvement qui a pris son origine à la fin du siècle précédent et qui plonge ses racines dans l'histoire sociale et culturelle de l'Occident. Les grands musées des capitales ne constituent que la partie la plus visible du phénomène. Des villes secondaires comme Birmingham, Neuchâtel ou Swansea, au pays de Galles, Chicago, Boston et même Salem, sur la côte est des Etats-Unis, ont également leur musée d'histoire naturelle ${ }^{4}$. Dans l'hémisphère sud, Melbourne, Christchurch et Buenos Aires rivalisent d'efforts pour leurs musées ${ }^{5}$.

Intéressante en soi, l'histoire des musées et des cabinets d'histoire naturelle contribue à éclairer l'histoire des sciences, de même que l'histoire de l'éducation et l'histoire de la culture. Elle est indissociable de l'histoire de la classification des êtres vivants, activité au coeur de la biologie prédarwinienne. Comme l'ont montré nombre d'historiens, le musée et la collection constituent un état donné des théories taxonomiques, la disposition ou l'arrangement des spécimens reflétant les allégeances scientifiques des conservateurs ${ }^{6}$. L'histoire des musées appartient également à l'histoire de l'éducation dans la mesure où plusieurs collections se sont développées pour l'enseignement ou, du moins, dans le cadre d'institutions d'enseignement. En outre, les musées et les collections ont été au centre de l'évolution de la pédagogie moderne, axée sur l'expérience et l'objet ${ }^{7}$. Enfin, les musées ouvrent sur la culture des perspectives d'un grand intérêt. Cela est vrai autant des cabinets de curiosités qui se multiplient, de la Renaissance à la fin de l'Ancien régime, que des musées publics ou privés qui apparaissent à compter

3 Camille Limoges, «The Development of the Muséum d'histoire naturelle of Paris, c. 18001914», Robert Fox et George Weisz, eds., The Organization of Science and Technology in France, 1808-1914 (Cambridge, 1980), 211-240; William T. Stearn, The Natural History Museum at South Kensington (Londres, 1981) et A. E. Gunther, A Century of Zoology at the British Museum (Londres, 1975); Phillip Kopper, The National Museum of Natural History (New York, 1982).

4 C. J. Schönburg-Hartenstein, Josef Anton Nagel: Ein Direktor des Physikalischen Kabinettes (Vienne, 1987); C. Dufour et J.-P. Haenni, Le Musée d'histoire naturelle de Neuchâtel (Hauterive, 1985); Sally G. Kohlstedt, «From Learned Society to Public Museum: the Boston Society of Natural History», A. Oleson et J. Voss, eds., The Organization of Knowledge in Modern America, 1860-1920 (Baltimore, 1979), 386-406; Ralph Dexter, «The Impact of Evolutionary Theories on the Salem Group of Agassiz Zoologists», Essex Institute Hist. Collections, 115 (1979): 144-171 et W. M. Whitehill, The East India Marine Society and the Peabody Museum of Salem (1949).

5 Sheets-Pyenson; S. G. Kohlstedt, «Australian Museums of Natural History: Public Priorities and Scientific Initiatives in the 19th Century», Historical Records of Australian Science, 5 (1983): 61-82.

6 On se rappellera les idées développées sur ce thème par Michel Foucault, Les mots et les choses (Paris, 1966), 140ss. Dans la veine proprement historique, Mary P. Winsor, Starfish, Jellyfish, and the Order of Life (New Haven, 1976), en particulier le chapitre 6; François Dagognet, Le catalogue de la vie (Paris, 1970); Paul Lawrence Farber, The Emergence of Ornithology as a Scientific Discipline, 1760-1850 (Londres, 1982), en particulier le chapitre 4.

7 S. G. Kohlstedt, «Museums on Campus: a Tradition of Inquiry and Teaching», K. Benson et al., eds., The Development of American Biology (Philadelphie, 1988), 15-47, et «International Exchange and National Style: a View of Natural History Museums in the United States», N. Reingold et M. Rosenberg, eds., Scientific Colonialism. A Cross-Cultural Comparison (Washington, 1987), 167-190. 
de $1800^{8}$. Toujours, la fréquentation du musée est signe de distinction sociale, preuve que l'on a du goût, de l'éducation et des loisirs, en même temps qu'une position à tenir et peut-être aussi quelqu'ambition à satisfaires.

Les historiens des mentalités et de la culture ont fait remarquer que la raison d'être et le fonctionnement d'un musée, avec ses opérations d'acquisition, d'inventaire, d'échange, de thésaurisation, etc., mimant les échanges économiques et l'accumulation capitaliste, épousaient tout à fait les goûts et les aspirations d'un public de possédants. Le succès des musées d'histoire naturelle au XIXe siècle s'explique également par la popularité de la théologie naturelle, l'amour que porte à la nature une population de plus en plus citadine et le prestige de la science, assuré par le progrès technique et l'essor économique ${ }^{10}$. Les musées et les collections peuvent refléter des aspirations et des intérêts fort divers, aussi bien scientifiques que sociaux.

Qu'en a-t-il été au Québec au siècle dernier? Que peuvent nous révéler du style de vie et des mentalités du XIXe siècle les collections d'histoire naturelle? À quelles aspirations répondaient les musées d'histoire naturelle créés entre 1824 et le tournant du XXe siècle (tableau 1)?

Des recherches récentes, dont nous faisons ici la synthèse, permettent de répondre à ces questions et tendent à montrer que l'histoire naturelle constituait un sujet d'intérêt pour un public beaucoup plus vaste que celui, fort étroit, des savants et des pédagogues. Comme nous le verrons, on distingue trois «vagues» successives dans le développement des musées d'histoire naturelle au Québec: celle des premiers musées, souvent associés à des sociétés d'amateurs, celle des musées d'enseignement, et enfin, celle des musées «d'État». Cette séquence de développement, tout à fait comparable à ce qui s'est déroulé en Europe et ailleurs en Amérique du Nord, indique que le Québec était relativement perméable aux courants scientifiques et culturels étrangers.

8 Krzysztof Pomian, Collectionneurs, amateurs et curieux. Paris-Venise: XVIe-XVIIe siècle (Paris, 1987); Lorraine J. Daston, «The Factual Sensibility», Isis, 79 (1988): 452-470; David E. Allen, The Naturalist in Britain. A Social History (Londres, 1976); Lynn Barber, The Heyday of Natural History (New York, 1980), en particulier le chapitre 11.

9 Pierre Bourdieu a analysé, du point de vue sociologique, le jeu de la distinction dans la fréquentation des musées. L'amour de l'art (Paris, 1965) et La distinction. Critique sociale du jugement (Paris, 1979), 307-309.

10 Allen; Barber; Joseph Kastner, A Species of Eternity (New York, 1977); Charlotte M. Porter, The Eagle's Nest: Natural History and American Ideas, 1812-1842 (University, 1986). Le thème du progrès technique est exploré dans le contexte des expositions universelles par Paul Greenhalgh, Ephemeral Vistas. The Expositions universelles, Great Exhibitions and World's Fairs, 1851-1939 (Manchester, 1988). 
TABLEAU 1

Musées et cabinets au XIXe siècle

\begin{tabular}{|c|c|c|}
\hline Type/titre & Création & Conservateur \\
\hline $\begin{array}{l}1 \text { - Musées de sociétés savantes } \\
\text { Literary and Historical Society of Quebec } \\
\text { Natural History Society of Montreal } \\
\text { Entomological Society of Ontario (Montreal) } \\
\text { Institut canadien de Québec }\end{array}$ & $\begin{array}{l}1824 \\
1827 \\
1872 \\
1876\end{array}$ & $\begin{array}{l}\text { J. Whiteaves } \\
\text { W. Couper }\end{array}$ \\
\hline $\begin{array}{l}\text { 2-Institutions d'enseignement } \\
\text { Université Laval - botanique } \\
\text { - zoologie } \\
\text { - géologie }\end{array}$ & $\begin{array}{l}1862 \\
1860 \\
1860\end{array}$ & $\begin{array}{l}\text { O. Brunet, prêtre } \\
\text { F.-X. Bélanger } \\
\text { C.-E. Dionne } \\
\text { T. S. Hunt } \\
\text { J.-C. K. Laflamme }\end{array}$ \\
\hline $\begin{array}{l}\text { McGill (Redpath Museum) } \\
\text { Collège Saint-Laurent } \\
\text { Séminaire de Sherbrooke } \\
\text { Collège de Lévis } \\
\text { École Jacques-Cartier } \\
\text { Collège de Montréal } \\
\text { Collège de Sainte-Anne } \\
\text { Collège Sainte-Marie } \\
\text { Couvent d'Hochelaga } \\
\text { Institut des Sourds-Muets } \\
\text { Macdonald College (Lyman) } \\
\text { Séminaire de Chicoutimi } \\
\text { Séminaire de Saint-Hyacinthe } \\
\text { Séminaire de Nicolet } \\
\text { Séminaire de Sainte-Thérèse } \\
\text { Collège Bourget } \\
\text { Collège de Joliette } \\
\text { Collège de Terrebonne }\end{array}$ & $\begin{array}{r}1881 \\
1880 \\
\text { ca. } 1900 \\
1914 \\
1878 \\
1878 \\
1871 \\
1876 \\
\text { ca. } 1890 \\
1889 \\
\text { ca. } 1874\end{array}$ & $\begin{array}{l}\text { W. Dawson } \\
\text { R. P. J.-C. Carrier } \\
\text { P.-A. Bégin, prêtre } \\
\text { H.-A. Verreau, prêtre } \\
\text { J. Moyen, prêtre } \\
\text { R. P. Rottot, S.J. } \\
\text { Frère Crête } \\
\text { V.-A. Huard, prêtre } \\
\text { F.-X. Burque, prêtre } \\
\text { T. Moreault, prêtre }\end{array}$ \\
\hline $\begin{array}{l}\text { 3- Musées publics } \\
\text { Musée Del Vecchio } \\
\text { Musée de Pierre Chasseur } \\
\text { Musée de la Commission géologique } \\
\text { Musée de l'Instruction publique }\end{array}$ & $\begin{array}{l}1824 \\
1824 \\
1842 \\
1886\end{array}$ & $\begin{array}{l}\text { T. Del Vecchio } \\
\text { P. Chasseur } \\
\text { E. Billings } \\
\text { D.-N. Saint-Cyr }\end{array}$ \\
\hline $\begin{array}{l}\text { 4- Collections particulières } \\
\text { Léon Provancher, prêtre } \\
\text { Alexandre Lechevallier } \\
\text { Cornélius Krieghoff } \\
\text { G. J. Bowles } \\
\text { Rév. T. Fyles } \\
\text { J.-A. Crevier, médecin } \\
\text { James M. LeMoine } \\
\text { David Roy, juge } \\
\text { É. Glackmeyer, notaire } \\
\text { Gustave Chagnon } \\
\text { Germain Beaulieu } \\
\text { C.-E. Dionne } \\
\text { T. Bédard, notaire } \\
\text { D.-N. Saint-Cyr } \\
\text { P. Lemay, prêtre } \\
\text { Denis-Benjamin Papineau }\end{array}$ & $\begin{aligned} 1860-1892 \\
1860 \\
\text { ca. } 1860 \\
\text { ca. } 1870 \\
1890-1910 \\
1859-1889 \\
1870-1900 \\
\text { ca. } 1860 \\
\\
1890- \\
1890- \\
1880-1925 \\
\text { ca. } 1887 \\
1870-1899 \\
-1918\end{aligned}$ & $\begin{array}{l}\text { Diverses } \\
\text { Ornithologie } \\
\text { Entomologie } \\
\qquad " \prime " \\
\text { Diverses } \\
\text { Ornithologie } \\
\text { Botanique " } \\
\text { Entomologie } \\
\text { Ornithologie } \\
\text { Botanique } \\
\text { Diverses } \\
\text { Botanique } \\
\text { Minéralogie }\end{array}$ \\
\hline
\end{tabular}




\section{1 - L'ÉVEIL DU PUBLIC ET LES PREMIERS MUSÉES (1824-1860)}

Tout comme la multiplication des journaux, des bibliothèques et des sociétés culturelles, la création des premiers musées et cabinets d'histoire naturelle est liée à l'apparition d'un public instruit et citadin ${ }^{11}$. À compter de 1820 , les cercles culturels se développent à Montréal et à Québec. Il s'y manifeste un véritable esprit encyclopédique, aucune discipline n'échappant à la curiosité du public.

En effet, on cultive les sciences au même titre que les arts ou la littérature, et l'histoire naturelle est la science de prédilection à cette époque. Ici encore, la bourgeoisie du Bas-Canada ne se distingue pas du public anglais ou américain dans sa préférence pour l'étude de la géologie, de la flore et de la faune ${ }^{12}$. Cet engouement pour l'histoire naturelle, comme on le notait plus haut, tient à des causes nombreuses. Beaucoup d'amateurs puisent dans l'étude des oeuvres de la «divine Providence» des arguments qui confortent le sentiment religieux. La vogue de la théologie naturelle, renouvelée au XVIIIe siècle par Bernardin de Saint-Pierre et William Paley, ne se dément pas. «L'étude de la nature faite au point de vue chrétien, écrit Mgr Laflèche, l'évêque ultramontain de Trois-Rivières, n'est pas seulement utile pour les choses du temps, mais nous élève encore vers Dieu en nous parlant avec tant d'évidence et d'éloquence de sa puissance.» ${ }^{13}$ Certains amateurs sont plus sensibles à la beauté des spécimens naturels et cultivent l'histoire naturelle comme les beaux-arts ou la littérature, la veine romantique trouvant ici à s'épancher. D'autres encore ont des intérêts plus pragmatiques. C'est le cas des ingénieurs civils ou de ceux qui se préoccupent d'agriculture «scientifique». Plusieurs des premiers relevés de la géologie canadienne sont l'oeuvre d'ingénieurs travaillant à la construction des routes et des canaux dans le Haut et le Bas-Canada ou au tracé des frontières. En agronomie, même si les progrès sont dus surtout aux chimistes, l'histoire naturelle joue un rôle important dans la connaissance des espèces utiles et nuisibles.

Les médecins s'intéressent également à l'histoire naturelle. Cette discipline fait partie de leur formation et de leur pratique car beaucoup de théories médicales de cette époque privilégient les éléments du milieu

11 R. A. Jarrell, «The Rise and Decline of Science at Quebec, 1824-1844», Histoire sociale Social History, 10 (1977): 77-91 et «The Social Functions of the Scientific Society in NineteenthCentury Canada», R. A. Jarrell et A. Roos, eds., Critical Issues in the History of Canadian Science (Thornhill, 1983), 31-44; Ginette Bernatchez, «La Société littéraire et historique de Québec, 1824-1890», Revue d'histoire de l'Amérique française, 35,2 (septembre 1981): 179-192; Marcel Lajeunesse, Les Sulpiciens et la vie culturelle à Montréal au XIXe siècle (Montréal, 1982); Yvan Lamonde, "Les associations au Bas-Canada: de nouveaux marchés aux idées», Histoire sociale/Social History (1975): 361-370.

${ }_{12}$ Allen; Barber; S. Sheets-Pyenson, «Popular Science Periodicals in Paris and London: the Emergence of Low Scientific Culture, 1820-1875», Annals of Science, 42 (1985): 549-572.

${ }_{13}$ Archives du Petit Séminaire de Chicoutimi (APSC): Fonds Provancher; lettre de Mgr Laflèche à l'abbé Léon Provancher, 3 novembre 1885 . 
naturel dans l'étiologie des maladies. La Société médicale de Québec, fondée en 1826, accorde une large place à l'histoire naturelle dans ses séances et dans son organe officiel, le Journal de médecine de Québec ${ }^{14}$. Les règlements précisent que la Société s'intéresse à la «Natural History, Botany, Chemistry, Pharmacy, Materia Medica, Physic, Surgery, Anatomy, Physiology, Medical Jurisprudence [...]», etc. ${ }^{15}$ En 1828, un membre en vue de la Société, le docteur François-Xavier Tessier, tente sans succès de lancer le Journal des sciences naturelles de l'Amérique du Nord ${ }^{16}$. À Montréal, le docteur Jean-Baptiste Meilleur se distingue en publiant quelques notes scientifiques dans la Bibliothèque canadienne et en acceptant de donner un cours de géologie au sein de la Natural History Society en $1842^{17}$.

On trouve aussi de nombreux naturalistes amateurs parmi les chasseurs sportifs. Les officiers britanniques de la garnison de Québec et leurs amis se distinguent particulièrement dans ce domaine et ne sont pas étrangers à la création de l'Association for the Protection of Game and Fish en $1859^{18}$. Fixé dans la vieille capitale de 1854 à 1862, Cornelius Krieghoff, qui fait partie de ce cercle et peint pour ses amis plusieurs scènes de chasse, collectionne les plantes et les oiseaux. Il appartient également à une petite société d'entomologie, filiale locale de l'Entomological Society of Canada. Un autre membre de cette société, William Couper, publie à Montréal, à compter de 1881, la revue Canadian Sportsman and Naturalist, qui, comme son titre l'indique, s'adresse à un double public. Encore à la fin du siècle, les ouvrages de Henri de Puyjalon, inspecteur des pêcheries et de la chasse de la province, répondent aussi bien à la curiosité du chasseur qu'à celle du naturaliste ${ }^{19}$.

Or, on ne fait pas d'histoire naturelle sans collections. L'omniprésence de celles-ci montre bien que l'étude de l'histoire naturelle fait partie du style de vie de la bourgeoisie canadienne. En 1824, Lady Dalhousie, épouse du gouverneur général, fait don de sa collection de plantes canadiennes à la Literary and Historical Society of Quebec (LHSQ), nouvellement organisée. Cette collection avait vraisemblablement été constituée lors d'herborisations dans les environs de la ville en compagnie de son amie, Harriet Sheppard ${ }^{20}$, épouse de William Sheppard, riche marchand de bois et naturaliste fervent. Celui-ci avait

\footnotetext{
14 Othmar Keel et Peter Keating, "Autour du Journal de médecine de Québec (1826-1827)», Critical Issues in the History of Canadian Science, 101-134.

15 Journal de médecine de Québec, 2 (1827): 108.

16 «Prospectus», La Minerve, 20 mars 1828

17 «Analyse de l'eau saline de l'Assomption», Bibliothèque canadienne, 2 (1826): 142 et 199. APSC: Fonds Provancher, lettre de Meilleur à Provancher, 2 juin 1869.

18 Vianney Legendre, «Limnology in Quebec», David G. Frey, ed., Limnology in North America (Madison, 1963), 495-519.

${ }_{19}$ Histoire naturelle à l' usage des chasseurs canadiens et des éleveurs d'animaux à fourrures (Québec, 1900).

20 James M. LeMoine, L'Album du touriste (Québec, 1872), 84.
} 
rassemblé dans sa villa de Woodfield, à Sillery, un petit musée d'histoire naturelle, des volières et des serres. Ce musée était pourtant moins réputé que la collection ornithologique créée par son voisin, James Macpherson LeMoine, dans son manoir de Spencer Grange ${ }^{21}$.

Les aristocrates et les riches marchands d'origine britannique ne sont pas les seuls à collectionner les spécimens d'histoire naturelle. Le notaire Louis-Édouard Glackmeyer, de Québec, s'intéresse à la météorologie et dresse un herbier dont il fait profiter son neveu, l'abbé LouisOvide Brunet, futur professeur de botanique à l'Université Laval ${ }^{22}$. Un autre botaniste amateur, le juge Louis-David Roy, avait dans sa jeunesse collectionné les plantes indigènes. En 1860, il fournit une liste d'espèces de la région du lac Saint-Jean à l'abbé Léon Provancher qui prépare sa Flore du Canada ${ }^{23}$. Le notaire Thomas Bédard, de Lotbinière, rassemble également un herbier considérable ${ }^{24}$.

Outre les collections privées, qui ne cessent de se multiplier tout au long du XIXe siècle, il existe des musées ouverts au public. Les premiers apparaissent simultanément à Montréal et à Québec en 1824. Il s'agit du Musée italien de Thomas Del Vecchio et du Musée de Pierre Chasseur.

En août 1824, Del Vecchio annonce qu' «après beaucoup de soins, de voyages et de dépenses», qui lui ont permis de rassembler une collection de quadrupèdes, d'amphibiens, d'oiseaux et de poissons, plus quelques curiosités comme des figures de cire et des artefacts amérindiens, il convie la population à son nouveau Museo italiano ${ }^{25}$. Le musée est installé dans l'auberge que tient Del Vecchio sur la place du VieuxMarché à Montréal. L'affaire n'est pas un succès: dans un avis à la population paru le 20 janvier 1826, le propriétaire annonce qu'il fermera le musée et dispersera les collections si l'intérêt du public ne se ravive pas. Del Vecchio n'a pas le temps de mettre sa menace à exécution: il meurt le 5 mai suivant et son musée sombre presque aussitôt dans l'obscurité.

L'entreprise de Pierre Chasseur dure un peu plus longtemps. Doreur et sculpteur de son état, Chasseur n'a guère de titres scientifiques. Cependant, il réussit à rassembler une collection assez considérable de spécimens naturels, des oiseaux surtout, dans sa maison de la haute

21 Roger Le Moine, Un Québécois bien tranquille (Québec, 1985), 123.

22 APC: Mg 24, I 94, Fonds L.-É. Glackmeyer. APSC: Fonds Provancher; lettre de Glackmeyer à Provancher, 24 avril 1865.

23 Québec, 1862. APSC: Fonds Provancher, lettre de Roy à Provancher, 30 avril 1860.

24 APSC: Fonds Provancher, lettre de Auguste Bédard à Provancher, 13 décembre 1887. Jean-Jacques Lefebvre, «Les évadés du notariat: Thomas Bédard, notaire et botaniste de Lotbinière. Son homonyme, Thomas Bédard de l'Assomption», Revue du notariat, 76,3 (1973): 118 133.

25 Canadian Spectator, 21 août 1824. 
ville de Québec ${ }^{26}$. Une campagne assez vigoureuse dans les journaux ne lui vaut qu'une popularité passagère auprès du public, mais elle attire sur son entreprise l'attention de quelques hommes politiques influents, dont John Neilson et Louis-Joseph Papineau ${ }^{27}$. Rapidement aux prises avec des difficultés financières, Chasseur obtient, grâce à l'intervention de ses amis, deux prêts de la Chambre d'Assemblée, garantis par la collection. Les raisons invoquées pour appuyer l'oeuvre de Chasseur sont intéressantes. En effet, on voit nettement se dessiner dans les propos des députés et de ceux qui sont appelés à témoigner devant le Comité de l'éducation de la Chambre, l'intention de faire de la collection de Chasseur le point de départ d'un musée «national» répondant à la fois aux besoins de la science, de l'enseignement et de l'éducation populaire. L'abbé Jérôme Demers, professeur de science et de philosophie au Séminaire de Québec, déclare qu'il s'agit d'une «collection extrêment précieuse» et «un superbe commencement dans un Cabinet d'Histoire Naturelle» ${ }^{28}$. En Chambre, un député plaide la cause du musée de Chasseur en rappelant ce que font d'autres gouvernements en faveur de semblables institutions:

Le Musée Chasseur est la première collection qui ait été formée dans ce pays nouveau; et, comme le célèbre musée de Peale à New York, il est sous le coup d'une saisie. Songez, messieurs, au muséum britannique qui, de temps à autre, a reçu de magnifiques additions. En France, on ne croit jamais mieux employer les deniers publics qu'en encourageant l'accroissement des collections et de tout ce qu'il y a de mieux, d'admirable dans la nature et dans les arts: les collections particulières y trouvent incessament un acquéreur dans la munificence publique. ${ }^{29}$

Ces échanges montrent que l'on est plus enclin au Bas-Canada à suivre l'exemple britannique, où l'État accepte de prêter main-forte à l'initiative privée dans la création de musées nationaux, que la tradition américaine du strict laisser faire ${ }^{30}$. Même s'il s'inspire de l'exemple des musées américains "commerciaux», tels ceux de Charles Willson Peale, à Philadelphie, et de Phineas T. Barnum ${ }^{31}$, le Musée Chasseur tend à

26 Un inventaire de Meilleur, réalisé à la demande du Comité de l'éducation du Bas-Canada, précise que le musée renfermait notamment «soixante-et-quinze quadrupèdes, quarante reptiles, et environ cinq cent oiseaux». Cet inventaire détaillé fut publié dans le Journal de la Chambre, Appendice 0.0 , en 1836, ainsi que dans Le Canadien, 8 avril 1836. R. Duchesne, «Magasin de curiosités ou musée scientifique? Le musée d'histoire naturelle de Pierre Chasseur à Québec (1824-1854)», Scientia canadiensis, 7,2 (1983): 59-79.

27 APC: MG 24 B2, 1: 627; lettre de Papineau à sa femme, 13 mars 1826.

28 Journal de la Chambre, appendices et témoignages, 5 décembre 1831. 240-241.

Cité par Isidore Lebrun, Tableau statistique et politique des deux Canadas (Paris, 1833),

30 Kohlstedt, «International Exchange...», 171

31 C. C. Sellers, Mr. Peale's Museum: Charles Willson Peale and the First Popular Museum of Natural Science and Art (New York, 1980); Toby Appel, «Science, Popular Culture and Profit: Peale's Philadelphia Museum», Journal of the Society for Bibliography of Natural History, 9, 4 (1980): 619-634. Avant de créer le cirque moderne, Barnum a d'abord été homme de musée, 
devenir un musée public provincial. En 1836, le gouvernement du BasCanada prend officiellement possession des collections de Chasseur et les installe dans l'édifice où siège la Chambre. Malheureusement, le projet d'un musée public est contrecarré par les événements de 1837 et de 1838. Compromis, Chasseur est arrêté à deux reprises et perd ses protecteurs à l'Assemblée. Le nouveau gouvernement du Canada-Uni abandonne les collections à la LHSQ.

Avec le Musée Chasseur disparaît le seul véritable musée public créé dans la première moitié du XIXe siècle. Toutefois, le public n'est pas entièrement privé car il peut visiter, au moins à certaines conditions, les musées des sociétés littéraires et scientifiques. Quelques-uns sont plus éphémères encore que les musées de Del Vecchio et de Chasseur. C'est le cas, par exemple, des collections rassemblées par les membres de la section québécoise de l'Entomological Society of Canada. D'autres ne font guère de place à l'histoire naturelle dans leurs collections: les Mechanics' Institutes de Montréal et de Québec collectionnent plutôt les instruments scientifiques et les modèles de machines industrielles. Cependant, au moins deux sociétés, la Natural History Society of Montreal (NHSM) et la LHSQ, ouvrent leur musée à leurs membres et au public.

Fondée en 1827, la NHSM recrute principalement ses membres dans la société anglophone de Montréal. Les médecins y occupent une place prépondérante ${ }^{32}$. Pour ses membres et le public, la Société tient des séances mensuelles et organise des concours, mais son musée, installé dans l'édifice de la faculté de médecine de l'Université McGill, occupe bientôt l'essentiel de l'attention. Les dons s'accumulent. On remarque en particulier ceux de capitaines de vaisseau relâchant au port de Montréal et d'agents de la Compagnie de la baie d'Hudson. Comme Chasseur l'avait fait, on s'adresse à la Chambre d'Assemblée dès 1828 pour obtenir des fonds destinés à la bibliothèque et au musée. Le marchand James Leslie, membre de l'Assemblée, se fait le porte-parole de la NHSM. Après avoir pris soin de noter que la Société «a été fondée sur les principes les plus libéraux, toutes les classes de la société y étant admises» et «qu'il ne peut résulter que de très grands avantages de l'étude des richesses naturelles du pays», il fait valoir qu' «en procurant une collection soigneusement classée de minéraux, de plantes et d'animaux, la Société offrira des avantages inestimables à ceux qui étudient l'Histoire Naturelle, qui jusqu'à présent ont été forcés dans leurs

montreur de curiosités et de merveilles; Alexander, 50-51; Neil Harris, Humbug: the Art of P.T. Barnum (Boston, 1973).

32 Peter J. Bowler, «The Early Development of Scientific Societies in Canada», A. Oleson et S. C. Brown, eds., The Pursuit of Knowledge in the Early American Republic (Baltimore, 1976), 326-339; R. A. Jarrell, «The Social Functions...»; Stanley B. Frost, «Science Education in the Nineteenth-Century. The Natural History Society of Montreal, 1827-1925», McGill Journal of Education, 17,1 (1982): 31-43. 
recherches à se procurer l'assistance des personnes étrangères du pays" ${ }^{33}$. A deux reprises, en 1829 et en 1830, la Chambre vote quelques centaines de livres en faveur de la Société.

Alors que beaucoup de sociétés scientifiques disparaissent une fois éteints les enthousiasmes du début, la popularité de la NHSM ne connaît qu'une courte éclipse à la fin des années 1840 , grâce en partie à ses liens avec l'Université McGill, qui remontent à l'origine de la Société. À compter de 1855 , la NHSM profite du nouveau souffle que donne William Dawson à l'université et, en particulier, aux études scientifiques. Dawson sera président de la Société à plusieurs reprises et c'est par son intermédiaire que celle-ci obtient en 1856, des gouverneurs de McGill, un terrain et un prêt pour construire un nouveau musée sur la rue de l'université ${ }^{34}$. Dans ses nouveaux quartiers, la NHSM connaît une période de popularité sans précédent. Ses séances et ses «lectures» sont très courues, non seulement par les amateurs de la ville, mais aussi par les étudiants et les professeurs de McGill, de même que par les scientifiques de la Commission géologique du Canada. La NHSM accueille également les savants étrangers, à l'occasion des congrès tenus à Montréal, de l'American Association for the Advancement of Science, en 1857 et en 1882, et de la British Association for the Advancement of Science, en 1884. Enfin, la Société prend sous sa responsabilité la publication d'une des plus anciennes revues scientifiques d'Amérique, le Canadian Naturalist and Geologist, créée en 1856 par Elkanah Billings. Institution culturelle de premier plan dans la vie montréalaise, la NHSM poursuivra ses activités jusqu'en 1925, emportée alors par des difficultés engendrées par la Première Guerre mondiale et par le déclin de popularité de l'histoire naturelle. Ses collections iront enrichir les musées de l'Université McGill.

À Québec, la LHSQ connaît une évolution assez semblable. Créée à l'instigation du gouverneur général lord Dalhousie et bénéficiant elle aussi des largesses de la Législature, elle se développe rapidement ${ }^{35}$. Dès les premières années, elle peut se targuer de compter parmi ses membres tous les citoyens éminents de la vieille capitale, canadiens et britanniques. Ses séances sont suivies avec intérêt et ses Transactions, qui commencent à paraître dès 1829 , forment un ensemble scientifique assez imposant ${ }^{36}$. Son musée s'enrichit grâce à la générosité de la Chambre et de ses membres. Madame Sheppard et lord Durham offrent

33 Journal de l'Assemblée (1828-1829): 59-60.

34 Frost, 40-41. Cet édifice sera le siège de la NHSM presque jusqu'à sa dissolution en 1925.

35 Jarrell, «The Rise and Decline of Science at Quebec, 1824-1844»; Bernatchez; Laura I. Bancroft, The Literary and Historical Society of Quebec, mémoire de maîtrise, Université Laval, 1950.

36 Les sciences naturelles occupent la première place dans les conférences données à la LHSQ entre 1829 et 1891 , devant l'histoire profane et les sciences physiques et mathématiques. Bernatchez, 186. 
des collections conchyliologiques et le capitaine Henry Bayfield, une collection minéralogique rassemblée au cours de ses expéditions hydrographiques dans le golfe Saint-Laurent et dans le Haut-Canada ${ }^{37}$. En 1841, la LHSQ recueille les collections de Chasseur. Décrivant le musée tel qu'il se présentait vers 1853 , un membre de la LHSQ écrivait en 1877 , sans doute avec un peu d'exagération:

Ce musée, alors le plus important de l'Amérique, faisait grand honneur à Québec. On y voyait des échantillons de presque toutes les productions naturelles du Canada, une collection d'oiseaux de l'Amérique, la plus belle de ce continent [...], une collection de tableaux et de portraits. ${ }^{38}$

Malheureusement, la LHSQ est sujette aux variations de la température politique dans la vieille ville de Québec. Les événements de 1837 ravivent les tensions entre les Britanniques et les Canadiens à un point tel que nombre de ces derniers quittent les rangs de la LHSQ. À compter de cette date, les amateurs francophones tenteront plutôt de se regrouper dans leurs propres sociétés littéraires ou scientifiques, telles l'éphémère Société canadienne d'études scientifiques et littéraires, l'Institut canadien de Québec ou la Société de géographie de Québec. Plus ou moins abandonnée par le public canadien, la LHSQ subit un nouveau coup lorsque le siège du gouvernement du Canada-Uni se déplace à Kingston en 1841. La Société perd alors plusieurs de ses membres qui appartenaient à la Législature ou à la fonction publique. Les éléments s'en mêlent également: en 1854, un incendie ravage l'édifice du Parlement, entraînant la perte du musée et de la bibliothèque. Les membres reconstituent l'un et l'autre, notamment avec l'aide du British Museum et de la Quebec Library Association, mais en 1862 un deuxième incendie réduit leurs efforts à néant. Malgré le zèle de quelques amateurs comme LeMoine et Couper, le musée de la LHSQ ne se remettra jamais de ce désastre. La LHSQ elle-même est sur son déclin. Institution d'envergure nationale à son origine, elle n'est plus, à compter du milieu du siècle, qu'une société locale dont le destin est lié à celui de la population anglophone de Québec. En 1891, faute de ressources, la LHSQ abandonne ses dernières collections d'histoire naturelle au High School de Québec.

Il existe au Bas-Canada un autre musée public d'une certaine importance; celui de la Commission géologique. Créée en 1842 par le gouvernement du Canada-Uni, la Commission géologique a pour responsabilité d'explorer les formations rocheuses du Bouclier canadien et de la chaîne des Appalaches et de dresser l'inventaire des ressources minérales du pays ${ }^{39}$. Organisée sur le modèle du Geological Survey

37 Joseph Bouchette, The British Dominions in North America (Londres, 1831), 1: 252.

38 Transactions, 1877-1878-1879, 39.

39 Morris Zaslow, Reading the Rocks. The Story of the Geological Survey of Canada, 1842 1972 (Ottawa, 1975); Suzanne Zeller, Inventing Canada. Early Victorian Science and the Idea of a Transcontinental Nation (Toronto, 1987), 51-112. 
britannique et des organismes américains créés dans plusieurs États, dirigée par Willam $E$. Logan, un géologue canadien formé sur le terrain au pays de Galles et en Pennsylvanie, la Commission devient rapidement une entreprise scientifique de première force. De Montréal, où elle a son quartier général et son musée, la Commission envoie chaque année sur le terrain des équipes de géologues de plus en plus nombreuses. Soucieux de se ménager des appuis parmi les membres de la Législature et dans le public, Logan veille à ce que le musée réponde non seulement aux besoins du service et des scientifiques qui le visitent, mais également à la curiosité du public. En 1844, il écrit au responsable du musée:

The object will be to produce an effect on the members [of Parliament]. With the same view, I must get a house or a set of rooms for our collection. Managing this, we must put our economic specimens conspicuously forward; and it appears to me that in the exhibition of these, large masses will make a greater impression on the mind than small specimens. ${ }^{40}$

L'expérience qu'acquièrent Logan et ses collaborateurs au musée de la Commission dans la présentation de minéraux et de fossiles au double public des experts et des néophytes leur est fort utile en 1851 lorsqu'ils sont chargés de présenter une collection tirée de leurs réserves à la Great Exhibition de Londres. Le succès remporté à cette occasion amène le gouvernement à confier régulièrement à la Commission géologique le soin de «monter» les collections canadiennes lors des expositions universelles qui suivent celle de Londres ${ }^{41}$. Musée scientifique et musée technique, fréquenté par les géologues, les paléontologistes et les ingénieurs miniers, le musée de la Commission est également un musée public, ouvert aux amateurs. Plus encore, il tend à devenir un véritable musée national, en raison du rôle que joue la Commission sur la scène internationale et du fait qu'elle relève du gouvernement du Dominion. Aussi comprend-on l'indignation des Montréalais lorsque le gouvernement fédéral décide de transférer à Ottawa le siège et le musée de la Commission géologique. Malgré les protestations du public et des édiles municipaux, les collections sont transportées dans la capitale en 1881 .

De 1824 à 1860 , tous les musées et cabinets d'histoire naturelle du Bas-Canada, à l'exception de celui de la Commission, sont des musées «privés», nés de l'intérêt de petits groupes d'amateurs pour la faune et la flore. Ils reflètent davantage le style de vie et les intérêts

\footnotetext{
181.

40 Cité dans Bernard J. Harrington, Life of Sir William E. Logan (Montréal, 1883), 180-

41 Logan et ses successeurs participent ainsi à celles de Paris, en 1855 et en 1867 , de Londres en 1862 et en 1886, de Philadelphie, en 1876, de Chicago en 1893, de Saint-Louis en 1904, etc. A. Shortt, «Canada Exhibited, 1851-1867», Canadian Historical Review 48 (1967): 353-365.
} 
culturels d'une classe aisée que des préoccupations «professionnelles». On peut souligner également le fait que nombre de ces musées, même s'ils accueillent parfois le grand public, demeurent des institutions «élitistes». Les collections de la NHSM et de la LHSQ appartiennent d'abord aux membres de ces deux sociétés et l'on ne trouve guère de traces d'efforts visant à «démocratiser» la fréquentation des salles. En 1830, lorsque l'Assemblée vote une subvention à la LHSQ, c'est à la condition «que le Musée et la Bibliothèque de la dite Société seront ouverts au Public» ${ }^{42}$. Ce caractère élitiste n'est pas propre aux sociétés dominées par les Britanniques: l'Institut canadien de Québec, qui inaugure un musée d'histoire naturelle en 1876, paraît tout aussi fermé ${ }^{43}$. L'étude de l'histoire naturelle ou même simplement la contemplation des spécimens est un plaisir qui paraît réservé à ceux qui en ont les moyens un «capital culturel», dirait Bourdieu. On ne trouve rien dans l'attitude des conservateurs ou des naturalistes de l'époque qui annonce le mouvement en faveur de l'éducation populaire qui va radicalement transformer les musées à la fin du XIXe siècle.

\section{2 - LES MUSÉES ET L'ESSOR DE L'ENSEIGNEMENT (1860-1890)}

À partir de 1840, le Bas-Canada développe son système d'éducation, système dont les collèges classiques forment l'épine dorsale ${ }^{44}$. Sur les deux rives du Saint-Laurent, un clergé bâtisseur multiplie les institutions. La création de l'Université Laval, en 1852, et la réforme de l'Université McGill, peu après, donnent une nouvelle dimension aux études supérieures dans la province.

Fondé en 1829, McGill College a connu des années difficiles. Le programme du baccalauréat ès arts, inspiré d'Oxford et de Cambridge, fait une trop large place aux humanités et à la théologie au goût des marchands et des industriels montréalais ${ }^{45}$. Aussi les inscriptions stagnent-elles et l'institution est aux prises avec des difficultés financières jusqu'au milieu du siècle. En 1855, le nouveau principal, le naturaliste John William Dawson, entreprend de réformer l'enseignement. Il ajoute des cours de science (zoologie, géologie, minéralogie, chimie, botanique, etc.) au programme du baccalauréat ès arts et inaugure un programme de baccalauréat ès sciences appliquées dès 1857. Un musée devient nécessaire. Pour remplacer les maigres collections d'histoire naturelle disparues dans un incendie en 1856, Dawson obtient de la famille Molson des dons qui permettent de construire un édifice destiné

\footnotetext{
42 Journal de l'Assemblée 1830, 201. La même condition s'applique à la NHSM.

43 Le musée est créé à la suite d'une souscription (APSC: Fonds Provancher; lettre de V. Bélanger à Provancher, 24 février 1876). En 1897, l'Institut remet ses collections à l'Université Laval (ASQ: Université 44, 16; lettre de Nazaire N. Ollivier à Mgr Hamel, 3 mai 1897).

${ }_{44}$ Claude Galarneau, Les collèges classiques au Canada français (Montréal, 1978), 25ss.

45 S. B. Frost, McGill University. For the Advancement of Learning (1801-1895) (Montréal, 1980), 149-176.
} 
à abriter notamment un nouveau musée. Au cours des années, McGill s'enrichit de nombreux dons. L'un des plus considérables est la collection de coquillages d'un naturaliste montréalais, le Révérend Philip Carpenter. Les professeurs et les étudiants peuvent également compter sur les collections de la NHSM et sur celles de la Commission géologique.

En 1881, Dawson ne perd pas de temps à regretter le déménagement de la Commission géologique à Ottawa. Il obtient de l'industriel montréalais Peter Redpath les fonds permettant la construction d'un splendide petit musée de style néo-classique au centre du campus de l'Universitée ${ }^{46}$. Le Musée Redpath est inauguré en 1882, juste à temps pour le congrès de la AAAS qui rassemble à Montréal des centaines de savants canadiens et américains. C'est un modèle de petit musée destiné à la recherche et à l'enseignement. Autour de l'impressionnant squelette du Megatherium, les collections zoologiques, paléontologiques et botaniques occupent les deux étages et la galerie du musée. Faute de pouvoir retenir les services d'un conservateur à plein temps, Dawson mobilise les professeurs de géologie, de botanique et de zoologie de McGill à titre de «conservateurs» des différentes sections. Cet arrangement crée cependant des difficultés et, après la mort de Dawson en 1899 , faute d'une direction ferme et de moyens suffisants, le Redpath Museum se développe lentement.

Créée en même temps que l'Université Laval elle-même, la Faculté des Arts de l'Université Laval doit développer l'enseignement supérieur des lettres, des humanités et des sciences, tout en couronnant par le diplôme de baccalauréat ès arts les études faites dans les collèges classiques de la province. Cependant, le projet se heurte au faible nombre de candidats aux études universitaires, hors des programmes de droit, de médecine ou de théologie, ainsi qu'à l'esprit d'indépendance des collèges. Il faudra plus de trente ans à l'Université Laval pour obtenir l'affiliation de tous les collèges de la province. La Faculté des Arts se voit longtemps réduite à n'offrir que des cours du soir et des conférences publiques.

Mais en voulant jouer le premier rôle dans le système d'éducation secondaire de la province, l'Université Laval s'est en quelque sorte condamnée à toujours payer d'exemple. On recrute pour la Faculté des Arts un bon noyau de professeurs: en sciences, on remarque l'abbé Thomas-Étienne Hamel, que le Séminaire de Québec envoie à Paris compléter la licence ès mathématiques, le minéralogiste américain Thomas Sterry Hunt, l'abbé Louis-Ovide Brunet, professeur de botanique,

46 S. Sheets-Pyenson, «Stones and Bones and Skeletons: the Origins and Early Development of the Peter Redpath Museum (1882-1912)», McGill Journal of Education, 17, 1 (1982): 49 62, et «Better than a Traveling Circus: Museums and Meetings in Montreal During the Early 1880s», Transactions of the Royal Society of Canada, série iv, 20 (1982): 499-518. 
et le docteur Hubert LaRue, professeur de chimie. On fait également des dépenses considérables pour les musées scientifiques, constitués à partir des collections déjà anciennes du Séminaire ${ }^{47}$. Dès 1858,1 ' $A n$ nuaire de l'université dresse un état avantageux des musées et des collections:

Le cabinet de Physique, maintenant à l'usage de la Faculté des Arts, est celui du Séminaire de Québec, qui a coûté au delà de $\$ 10,000$ piastres et qui est un des plus complets de l'Amérique. Cependant on a cru devoir y ajouter encore, et le professeur de Physique, actuellement en Europe, est autorisé à employer $\$ 4,000$ piastres à l'achat de nouveaux appareils. Quant au Musée de Minéralogie et de Géologie, il se compose des collections du Séminaire, qui renferment plus de 1,900 échantillons, et d'une autre faite pour l'Université par les messieurs de la Commission pour l'exploration géologique du Canada. Cette dernière comptera à peu près 2,000 échantillons. La Faculté des Arts possède plusieurs herbiers qui renferment chacun un très grand nombre d'échantillons. ${ }^{48}$

Ce n'est là qu'un début, car au cours des années suivantes et jusqu'à la fin du siècle, les autorités de l'Université Laval ne ménageront pas leurs efforts pour accroître et mettre en valeur les collections scientifiques. Continuellement enrichi, le Cabinet de physique permet aux professeurs de maintenir leur enseignement à la pointe de la discipline. Lors de conférences publiques, les instruments servent également à reproduire des expériences scientifiques, comme l'émission des rayons $\mathrm{X}$ en 1896, ou à faire connaître les dernières inventions du temps ${ }^{49}$.

À tour de rôle, les autres musées profitent du zèle ou de la curiosité scientifique des professeurs de l'universités0 ${ }^{50}$ La botanique connaît son heure de gloire avec l'abbé Brunet, de 1860 à 1870. Avec la collaboration de botanistes américains, Brunet enrichit l'herbier de spécimens nord-américains pour une étude qu'il prépare sur la flore canadienne. À compter de 1870, c'est sur la zoologie que se porte plutôt l'attention des professeurs. Le recteur Thomas-Etienne Hamel et François-Xavier Bélanger, conservateur du Musée de zoologie, font des achats considérables auprès de marchands canadiens, américains et européens. La

47 Dès 1806, l'abbé Jérôme Demers crée un musée scientifique. L'élément principal de ce musée fut probablement la collection minéralogique de l'abbé Haüy acquise en 1816 (ASQ: Séminaire 87).

48 Annuaire 1858-1859, 23.

49 Paul Carle, Le Cabinet de physique et l'enseignement des sciences au Canada français: le cas du Séminaire de Québec et de l'Université Laval, thèse de doctorat, Université de Montréal, 1986; P. Carle et R. Duchesne, «Modernisme et tradition: l'enseignement de la physique à Québec (1800-1920)», Protée, 16,3 (1988): 77-84; Yves Gingras, «La réception des rayons X au Québec: radiographie des pratiques scientifiques», Marcel Fournier, Yves Gingras et Othmar Keel, dir., Sciences et médecine au Québec (Québec, 1987), 69-86.

50 Pascale Gagnon, Sciences naturelles et muséologie au Canada français: le musée de l'Université Laval, mémoire de maîtrise, Université du Québec à Montréal, 1989. Paul Carle et Madeleine Dufresne, «Les musées scientifiques de l'Université Laval», Musées, 12,2 (1990): 6-13. 
vogue de l'histoire naturelle a donné naissance à un commerce international florissant, mais les échanges entre les naturalistes et les musées continuent d'alimenter à peu de frais la croissance des collections. En 1875, Hamel et Bélanger font paraître, dans le Journal entomologique de France, une note invitant les «naturalistes de bonne volonté» à échanger des spécimens avec l'Université Laval. Un premier inventaire des collections, paru la même année, nous apprend que celles-ci comprennent plus de 1300 oiseaux, une centaine de mammifères et plus de 12000 insectes, pour la plupart identifiés ${ }^{51}$. En 1882, CharlesEusèbe Dionne remplace Bélanger au Musée de zoologie. Ornithologue et taxidermiste de grande réputation, auteur de plusieurs ouvrages de vulgarisation en histoire naturelle, Dionne enrichira considérablement les collections de l'Université Laval jusqu'à sa mort en $1925^{52}$. Enfin, les musées de géologie et de minéralogie se développent sous la responsabilité successive de trois excellents professeurs: Thomas Sterry Hunt, l'abbé Hamel et l'abbé Joseph-Clovis Kemner Laflamme ${ }^{53}$. Géologue lui-même, l'abbé Laflamme veillera à faire ré-arranger périodiquement les collections paléontologiques canadiennes, réformes que rend nécessaires le progrès constant de la discipline, et entretiendra un circuit d'échanges avec le musée de la Commission géologique et des institutions étrangères comme la Smithsonian Institution de Washington, le Musée royal d'histoire naturelle de Bruxelles et le Muséum d'histoire naturelle de Paris.

L'exemple de l'Université Laval et du Séminaire de Québec n'est guère suivi avant 1870 . Si l'enseignement des mathématiques et de la physique se donne de manière convenable dans plusieurs collèges, il n'en va pas de même pour l'histoire naturelle, matière populaire, mais jugée «mineure» et laissée à la curiosité de l'élève. À Saint-Hyacinthe, par exemple, les élèves ont formé d'eux-mêmes une société d'histoire naturelle et «s'engagent à fournir au musée du Collège, après leurs heures d'études, des spécimens» ${ }^{54}$. Malgré tout, l'Université Laval maintient cette matière au programme de l'examen du baccalauréat et, au fur et à mesure que les collèges acceptent l'affiliation, l'enseignement de l'histoire naturelle se répand et s'élève. En 1874, l'Annuaire de l'Université Laval précise que le programme des deux années de Philosophie comporte au moins 120 leçons d'histoire naturelle: c'est presque autant que la physique et la chimie, même si cela ne représente que la moitié du temps consacré à l'étude de la philosophie. En 1880, la place de l'histoire naturelle dans le programme du baccalauréat se trouve consacrée lors du congrès qui réunit à Québec les représentants

51 Annuaire de l'Université Laval, 1875.

52 Victor Gaboriault, Charles-Eusèbe Dionne, naturaliste (La Pocatière, 1974).

53 René Bureau, «Le musée de minéralogie et de géologie de l'Université Laval», Naturaliste canadien, 76 (1949): 205-222.

54 Mgr C.-P. Choquette, Histoire du Séminaire de Saint-Hyacinthe (Montréal, 1911), 289. 
de quinze collèges ${ }^{55}$. Tout en refusant d'ajouter formellement au programme des éléments de zoologie, d'anthropologie et de physiologie suggérés par l'université, les membres du Comité des sciences approuvent l'état actuel de l'enseignement de l'histoire naturelle et vont même jusqu'à reconnaître «qu'il est désirable que les Collèges introduisent quelques notions des sciences ci-dessus mentionnées dans leurs cours d'études» ${ }^{56}$. Quelques manuels communs facilitent l'enseignement de l'histoire naturelle $\mathrm{e}^{7}$.

Un à un, les collèges emboîtent le pas au Séminaire de Québec et se dotent de musées d'histoire naturelle. Le Collège de Montréal, où l'enseignement des sciences a longtemps rivalisé avec celui du Séminaire de Québec, n'est pas le dernier à suivre le mouvement. Un sulpicien français, M. Jean Moyen, arrivé en 1858, ajoute à l'enseignement des mathématiques et de la physique celui de la botanique et de la géologie. Pour compléter ses cours, il organise le cabinet de physique et le musée d'histoire naturelle. Il rassemble en outre un herbier considérable ${ }^{58}$.

Le Collège de Saint-Laurent est une autre institution où l'on fait des efforts considérables pour la création et l'enrichissement d'un musée. Ici encore, l'initiative vient d'un prêtre français, le R. P. Joseph-C. Carrier, professeur de sciences et de philosophie. A partir de 1885, tous ses efforts vont au musée. «Jusque-là, écrit-il, le collège n'avait à proprement parler, que quelques oiseaux assez mal empaillés et fort détériorés, et une vingtaine d'oeufs d'oiseaux non classés.» ${ }^{59}$ Travailleur infatigable, Carrier rassemble en moins de dix ans plus de 80000 objets par des achats, des échanges et en recueillant des dons. La spécialisation n'est pas du goût du conservateur: le musée compte une collection numismatique, des milliers de gravures et de sculptures, une collection de plus de 1500 boutons, «don d'un jeune monomane», et même un «département d'indianeries» ${ }^{60}$. Toutefois, l'histoire naturelle occupe la place centrale: on trouve au musée des collections de mammifères, d'oiseaux, des reptiles, une collection ostéologique, des fossiles, etc. Carrier s'intéressant tout particulièrement à la botanique, l'herbier des plantes nord-américaines est considérable. Dans les autres branches de l'histoire naturelle, le conservateur a pu acquérir quelques collections

\footnotetext{
55 H.-J.-J.-B. Chouinard, Fête nationale des Canadiens français de 1880 (Québec, 1881).

56 ASQ: Université 99, 47. Rapport du Comité des sciences, juin 1880.

57 On dispose alors des Éléments de botanique (Québec, 1870), de Brunet, des Éléments de minéralogie et de géologie (Québec, 1881), de Laflamme, quatre fois réédité, et du Traité élémentaire de botanique (Québec, 1884), de l'abbé Provancher, «mis en rapport avec le programme de baccalauréat de l'Université Laval».

58 APSC: Fonds Provancher; lettre de Jean-Baptiste Meilleur à l'abbé Provancher, 16 mars 1869. Bernard Boivin, Survey of Canadian Herbaria, Université Laval (Québec, 1980), 64. Désigne l'herbier du Frère Louis-Marie.

9 R. P. J.-C. Carrier, «Le Musée du Collège Saint-Laurent», Revue canadienne, (octobre 60 Ibid., 609.
} 1896): 603 
privées importantes; la collection minéralogique du docteur J.-Alexandre Crevier, naturaliste amateur de Montréal, achetée de sa veuve en $1889^{61}$, des hyménoptères et des coquillages de l'abbé Provancher. En 1896, les collections ont pris une telle envergure que le Collège de SaintLaurent doit reloger le musée. Une souscription publique permet de faire construire un «édifice à huit côtés, de 144 pieds de périmètre, avec portique orné, de quatre étages avec galeries et dôme servant d'observatoire» ${ }^{62}$. On peut y voir une indication de l'importance que l'institution accorde au musée du père Carrier.

Les musées du Collège de Montréal et du Collège de Saint-Laurent ont peu d'égaux, mais presque tous les collèges ont leur collection d'histoire naturelle. En 1887, après enquête, l'abbé Provancher publie dans son Naturaliste canadien une liste d'une dizaine de maisons d'éducation possédant un musée ${ }^{63}$. Et encore le tableau n'est pas complet. Comme il le déplore lui-même, quelques collèges qui pourraient figurer dans la liste n'ont pas répondu à son appel. Néanmoins, l'article du Naturaliste stimule l'ardeur de quelques naturalistes amateurs car, deux ans plus tard, Provancher note avec plaisir que «les collèges de Lévis, de Rigaud, les couvents du Bon Pasteur, des Soeurs de la Charité de Québec, etc., ont commencé à s'occuper activement de la formation de musées.» ${ }^{64}$

L'éditeur du Naturaliste canadien n'est pas étranger à la multiplication des musées d'histoire naturelle. Naturaliste de grand talent, Provancher entretient dans les collèges tout un réseau de correspondants. L'un d'entre eux, l'abbé Pierre-Achille Bégin, est à l'origine de musées dans deux institutions où il enseigne successivement: le Séminaire de Sherbrooke (1879) et le Collège de Lévis (1889). Un autre disciple, le jeune abbé François-Xavier Burque, fait de même au Séminaire de SaintHyacinthe. Un troisième, l'abbé Victor-Alphonse Huard, crée le Musée du Séminaire de Chicoutimi. On trouve également des collections dans des institutions plus anciennes, comme le Collège de Sainte-Anne-dela-Pocatière ou le Séminaire de Nicolet, où l'abbé Thomas Moreault rassemble un herbier vers $1872^{65}$.

Pour ne pas être en reste sur les collèges classiques, d'autres institutions constituent également des collections d'histoire naturelle. À leurs couvents d'Hochelaga et de Sillery, les Soeurs de Jésus-Marie invitent l'abbé Provancher à donner quelques leçons d'histoire naturelle

\footnotetext{
61 APSC: Fonds Provancher; lettre de Carrier à Provancher, 25 novembre 1889; Jacques Rousseau, «Le docteur J.-A. Crevier», Annales de l'ACFAS, (1940): 173-271.

65 Omer Caron, «Herbier du Séminaire de Nicolet», Naturaliste canadien, 8-9,57 (1930):

182-183; Georges Courchesne, «Notes sur l'abbé Thomas-M.-O. Maurault (1839-1887)», Rapport de la Société canadienne d' histoire de l'Église catholique, 8 (1943-1944): 15-32.
} 
à leurs élèves et à initier les religieuses à la collecte et à la classification des spécimens ${ }^{66}$. Le principal de l'École normale Jacques-Cartier, l'abbé Hospice-A. Verreau, se charge lui-même de l'enseignement de l'histoire naturelle et constitue à cette fin un musée assez important ${ }^{67}$. Enfin, les communautés de frères enseignants qui étendent à la fin du siècle leur enseignement au niveau secondaire, comptent dans leurs rangs de bons naturalistes, en particulier parmi les frères venus de France. Le frère Rolland-Germain, é. c., originaire de Bourgogne, est un excellent taxonomiste qui initie le jeune frère Marie-Victorin à l'étude de la flore canadienne. Chez les Clercs de Saint-Viateur, le frère Florian Crête fera du Musée d'histoire naturelle de l'Institution des Sourds-Muets, à Montréal, un remarquable musée pédagogique, fondé sur le toucher et la vision, et inspiré du renouveau muséologique qui s'amorce aux ÉtatsUnis et en Europe après $1900^{68}$.

Pour acquérir des spécimens, les collèges et les écoles peuvent s'adresser à de nombreux marchands locaux et étrangers. Les établissements de A. E. Foote, à Philadelphie, de Cassino, à Salem, ou le Comptoir de conchyliologie de Paris sont célèbres et comptent plusieurs naturalistes canadiens parmi leurs clients ${ }^{69}$. Au Québec, des marchands comme William Couper et $\mathrm{A}$. Lechevallier réussissent à se maintenir grâce à la clientèle des maisons d'éducation. Naturaliste français établi à Montréal, Lechevallier compte parmi ses clients l'École normale Jacques-Cartier, le Séminaire de Québec, le Séminaire de Saint-Hyacinthe, etc. Il lui arrive de faire des expéditions de chasse aux spécimens en empruntant les fonds nécessaires auprès de ses clients: les commanditaires sont remboursés par des spécimens. Ainsi, par exemple, il parcourt la Côte Nord pour le compte des Oblats en 1868. En 1872 , l'Université Laval commandite un voyage semblable en Floride ${ }^{70}$. Les collèges peuvent également récupérer à l'occasion les collections de spécimens que les naturalistes possèdent en double et qu'ils sont prêts à vendre à meilleur marché. Par exemple, le Collège de Lévis acquiert en 1889 des collections entomologiques de l'abbé Provancher ${ }^{71}$. Mais les fonds sont rares et il faut surtout compter sur les échanges et les dons. Heureusement, des organismes comme la Commission géologique et la Smithsonian Institution offrent gratuitement aux établissements d'éducation des collections «pédagogiques».

66 APSC: Fonds Huard, 41-3; lettre de Provancher à l'abbé Huard, 12 juin 1880.

67 Université du Québec à Montréal (UQAM), Service des archives: Fonds de l'École normale Jacques-Cartier; 2P14/E,1, «Catalogue des différentes collections de l'École normale JacquesCartier», 1860, mss. 114 p.; Documents de la session (1878-1879) (42 Victoria): 180-181.

68 F. Crête, c.s.v., «L'histoire d'un musée», Naturaliste canadien, 43,2 (1916): 26-32 et 66-70. Paul Carle et Pascale Gagnon, «Florian Crête, c.s.v. et le Musée éducatif de l'Institut des Sourds-Muets (1882-1970): la leçon de choses», mss. 1990.

69 S. G. Kohlstedt, «Henry A. Ward: the Merchant Naturalist and American Museum Development», Journal of the Society for the Bibliography of Natural History, 9,4 (1980): 647-661.

70 ASQ: Fonds Verreau 31, 379; lettre de J.-M. LeMoine à Verreau, 28 février 1869.

71 APSC: Fonds Provancher, lettre de l'abbé P.-A. Bégin à Provancher, 24 août 1889. 
La diversité des collections montre que chaque conservateur dispose d'une grande marge de manoeuvre. Il arrive souvent que les collections aient peu à voir avec les exigences du programme de l'Université Laval et dépendent davantage des intérêts personnels du conservateur. Les disciples de Provancher collectionnent surtout les insectes. L'abbé Moreault, l'abbé Moyen et le R. P. Carrier préfèrent la botanique. Dans plusieurs cas, la richesse des collections excède les besoins de l'enseignement des différentes branches de l'histoire naturelle, qui reste élémentaire. Les conservateurs sont donc libres de poursuivre leurs propres intérêts scientifiques, souvent avec l'encouragement des institutions qui pratiquent une politique de prestige à travers leurs collections d'histoire naturelle et leurs cabinets de physique.

Cette émulation entre les collèges est ancienne. Déjà en 1836, le fameux voyage de l'abbé Jean Holmes, chargé d'acheter des livres et des instruments en Europe pour le compte de plusieurs établissements, avait donné lieu à une vive compétition. En 1884, le R. P. Carrier note qu'un musée est toujours «une source d'intérêt et de plaisir pour le visiteur, non moins qu'un sujet de légitime satisfaction pour les professeurs et les élèves de l'établissement» ${ }^{72}$. La lutte est d'autant plus vive qu'elle est publique. En faisant connaître les résultats de son enquête dans le Naturaliste canadien, Provancher savait bien qu'il allait provoquer des rivalités: «Chacun s'est rendu compte de ce qu'il possède, écrit-il, et voit ses richesses étalées à côté de celles de ses voisins. C'est à qui maintenant montera plus promptement dans l'échelle et devancera ses rivaux.» ${ }^{73}$ Bien sûr, les collèges eux-mêmes font connaître la richesse de leurs musées en publiant dans leurs annuaires la liste des donateurs et le catalogue de leurs collections. Les expositions provinciales et internationales leur fournissent également l'occasion de rivaliser. Enfin, le surintendant de l'Instruction publique publie dans son rapport annuel un tableau statistique de la valeur des musées et collections des établissements de la province ${ }^{74}$. Chaque collège doit donc s'efforcer de faire bonne figure, ce qui explique les sommes considérables que plusieurs consacrent aux musées.

Le mouvement ralentit pourtant à compter de 1890. Le «plein» est-il fait? Les collèges s'estiment-ils suffisamment pourvus de collections d'histoire naturelle pour soutenir la comparaison avec le Séminaire de Québec? Chose certaine, l'enseignement même de l'histoire naturelle dans le programme du baccalauréat marque le pas. En effet, au deuxième congrès des collèges, qui a lieu en 1890 et en 1891 , on convient de retirer l'histoire naturelle de la liste des matières sujettes à l'examen commun du baccalauréat. Chaque collège pourra continuer

\footnotetext{
Annuaire du Collège de Saint-Laurent (1884): 61.

Naturaliste canadien, 16,9 (1887): 135.

4 Voir, par exemple, les Documents de la session (1877-1878) (41 Victoria).
} 
d'enseigner l'histoire naturelle à sa convenance et faire passer ses propres examens aux étudiants. Plusieurs institutions choisissent alors de s'en tenir au strict minimum, c'est-à-dire aux sujets abordés sommairement dans les Éléments de minéralogie, de géologie et de botanique, de l'abbé Laflamme.

Avec le retrait de l'histoire naturelle de la liste des matières «universitaires», les musées des collèges perdent l'une de leurs principales raisons d'être et, à compter de 1890 , leur sort est de plus en plus nettement lié à l'intérêt ou à la bonne volonté des professeurs de science de chaque institution. Or, les jeunes ecclésiastiques auxquels on confie ces cours quittent souvent l'enseignement pour le ministère après quelques années: entre 1880 et 1890 , le Collège de l'Assomption change ainsi quatre fois de maître de sciences. Aussi ne se surprend-on pas de voir plusieurs collections disparaitre au tournant du siècle. Quand MarieVictorin et ses collaborateurs entreprennent leur étude de la flore laurentienne, ils ne trouvent souvent plus dans les herbiers des collèges que des vestiges des collections du XIXe siècle.

\section{3 - L'INTERVENTION DE L'ÉTAT À L'ÂGE DU MUSÉE PUBLIC (1880-1900)}

Dans le dernier quart du XIXe siècle, l'histoire des musées d'histoire naturelle prend un tournant. Un mouvement international se développe en faveur de l'éducation populaire à travers les musées et les collections. Ce mouvement s'explique à la fois par l'évolution des mentalités, qui va dans le sens d'une «démocratisation du savoir», et par le fait que beaucoup de musées privés sont devenus trop lourds à supporter pour des sociétés d'amateurs et des institutions d'enseignement. Afin d'obtenir l'appui du public, on multiplie les programmes éducatifs et les expositions destinées aux néophytes ${ }^{75}$. L'organisation physique même des musées se transforme: on réaménage les salles pour les rendre plus accueillantes aux écoliers et au public du dimanche, et les collections de recherche sont reléguées à la réserve, cédant la place à des arrangements de spécimens plus spectaculaires ou plus éducatifs. Ce mouvement s'accompagne d'une transformation du statut de nombreux musées privés qui sont pris en charge par les pouvoirs publics ${ }^{76}$.

Au Canada, le musée le plus sensible à ce mouvement est celui de la Commission géologique. Dans les années suivant son transfert à Ottawa, le Musée de la Commission géologique élargit substantiellement le champ de ses collections ${ }^{77}$. Le botaniste John Macoun, qui se

75 Kohlstedt, «International Exchange...», 175-180; Alexander, 50ss.

76 Cette évolution est commune aux grands musées des capitales d'Europe et aux musées «coloniaux»: Sheets-Pyenson, Cathedrals of Science ...; Kopper; Kohlstedt, "Australian Museums of Natural History...»; Douglas Sloan, «Science in New York City, 1867-1907», Isis, 71 (1980): 35-76.

77 En fait, c'est en 1877 que le Parlement d'Ottawa élargit par loi le mandat de la Commission, d'ailleurs rebaptisée «Geological and Natural History Survey of Canada». 
joint à la Commission en 1882, veille à rassembler des spécimens de l'histoire naturelle du territoire du Dominion, qui s'étend désormais d'un océan à l'autre. Les naturalistes de la Commission commencent également à étudier l'ethnologie des peuples amérindiens ${ }^{78}$. Avec le temps, sous la poussée du mouvement en faveur des musées publics et éducatifs, les collections de la Commission géologique donnent naissance au premier musée national du Canada. Dès 1900, le directeur de la Commission parle de son musée comme du «National Museum of Canada ${ }^{79}$ et l'année suivante, le gouvernement de Laurier annonce son intention de créer le Victoria Memorial Museum afin de loger et de mettre à la disposition du public les collections de la Commission géologique.

À Toronto, les pressions du public et des conservateurs amènent la Législature provinciale à créer, en 1912, le Royal Ontario Museum, qui recueille les collections scientifiques et ethnologiques de plusieurs petits musées de sociétés savantes et de collèges affiliés à l'Université de Toronto ${ }^{80}$.

Au Québec, le mouvement en faveur des musées publics ne trouve qu'un écho: la création par le gouvernement de la province du Musée de l'Instruction publique ${ }^{81}$.

Le projet d'un musée provincial d'histoire naturelle, consacré notamment à l'étude des espèces utiles ou nuisibles à l'agriculture, était relativement ancien. À compter de 1868, le Conseil de l'Agriculture de la province fait campagne pour la création d'un tel organisme, «le plus sûr moyen, écrit son secrétaire, d'enseigner d'une manière pratique les rapports qui existent entre l'homme et la création ${ }^{82}$. En 1875 , l'éditeur du Naturaliste canadien reprend le projet à son compte et achève d'y gagner Boucher de Boucherville, chef du gouvernement et ministre de l'Agriculture ${ }^{83}$. En 1877, Provancher cède une partie de ses collections d'insectes au département de l'Agriculture de la province. Par la même occasion, il devient «curateur» de celles-ci et rédacteur associé au Journal d'Agriculture, organe officiel du gouvernement ${ }^{84}$. Malheureuse-

\footnotetext{
78 William Waiser, «Canada on Display. Towards a National Museum, 1881-1911», Critical Issues in the History of Canadian Science, Technology and Medicine, 167-177, et The Field Naturalist. John Macoun, the Geological Survey and Natural Science (Toronto, 1989).

79 Cité par Waiser, «Canada on Display...», 173. Ce titre ne sera officiellement attribué au musée qu'en 1927.

${ }_{80}$ Lovat Dickson, The Museum Makers: the Story of the Royal Ontario Museum (Toronto, 1986); Lynn Teather, «For Educational Utility and Public Instruction: the University Museums of Victorian Toronto, 1842-1912», mss. 1989.

${ }_{81}$ Raymond Duchesne, Sciences, culture savante et pouvoir politique: le Musée de l'Instruction publique et l'histoire naturelle au Canada français, thèse de doctorat, Université de Montréal, 1984.

82 «Rapport du Secrétaire de la Chambre d'Agriculture», Documents de la session, 1869 (32 Victoria): 62-63.

83 Abbé Léon Provancher, «Si nous étions ministre», Naturaliste canadien, 7,2 (1875): 33 39 , et «Notre neuvième volume», Naturaliste canadien, 9,1 (1877): 4-5.

84 APSC: Fonds Provancher; lettre d'Ernest Gagnon à Provancher, 17 août 1877.
} 
ment pour lui, le projet d'un musée agricole est victime des événements politiques. Lorsque le «coup d'État» de Letellier de Saint-Just met fin au gouvernement de Boucher de Boucherville, Provancher, ultramontain farouche qui ne compte plus ses ennemis dans les deux partis à la Chambre, se voit retirer toutes ses charges.

Mais le projet d'un musée provincial d'histoire naturelle n'est pas tout à fait abandonné. Dominique-Napoléon Saint-Cyr, membre de l'Assemblée de 1875 à 1881 et naturaliste amateur, se glisse dans les bonnes grâces de Gédéon Ouimet, surintendant de l'Instruction publique, et se fait confier le soin d'ajouter aux collections entomologiques de Provancher un herbier canadien et quelques spécimens de la faune locale. Ses efforts aboutissent en 1886 à la création du Musée de l'Instruction publique et à sa nomination au poste de conservateur.

En dépit de son nom, le Musée n'est pas à proprement parler une institution d'éducation et ses collections ne sont destinées ni aux écoliers, ni au public en général. Les salles, dans une aile de l'édifice de l'Assemblée, ne sont pas aisément accessibles et, de toutes façons, le visiteur n'y trouverait qu'un herbier et des collections d'insectes classés selon les règles de la taxonomie - ce qui n'est guère utile à qui n'est pas spécialiste - , le tout soigneusement rangé dans des armoires hermétiques. Contrairement à ce qui se produit à cette époque dans beaucoup de musées d'histoire naturelle ouverts au public, on ne fait au Musée de l'Instruction publique aucun effort de vulgarisation.

Le rôle véritable du musée de Saint-Cyr est plutôt de constituer une réserve à même le produit des explorations du territoire du Québec. Elles sont nombreuses à cette époque car la bourgeoisie de Québec et les membres de l'Assemblée en particulier ont des visées expansionnistes sur le Moyen Nord, du Labrador à la baie James, en passant par la région du lac Saint-Jean ${ }^{85}$. La Société de géographie de Québec est particulièrement active dans ce mouvement en faveur de l'exploration et de l'exploitation de ces régions dont l'étendue et les ressources sont encore mal connues ${ }^{86}$. En fait, les conditions climatiques et le sol du Moyen Nord sont si mal connus que certains rêvent même d'un vaste mouvement de colonisation qui contrebalancerait l'exode des Canadiens français vers la Nouvelle-Angleterre. Plus nombreux sont ceux qui spéculent à long terme sur l'exploitation des forêts et des gisements miniers ou sur l'ouverture d'une route vers l'Ouest, ce qui permettrait au port de Québec de concurrencer celui de Montréal. Aussi voit-on le

85 Christian Morissonneau, La terre promise: le mythe du Nord québécois (Montréal, 1981); Gabriel Dussault, Le Curé Labelle. Messianisme, utopie et colonisation au Québec (1850-1900) (Montréal, 1983); Gérard Bouchard, «Une Nouvelle-France entre le Saguenay et la Baie-James: un essai de recommencement national au dix-neuvième siècle», Canadian Historical Review, 70,4 (1989): 473-495.

86 Christian Morissonneau, La Société de géographie de Québec (Québec, 1971). 
gouvernement provincial, qui n'hésite pas à faire siennes ces ambitions, multiplier les explorations du Moyen Nord.

Saint-Cyr lui-même explore la Côte Nord et le Labrador en 1882 et en 1885, mandaté par le gouvernement. Il en rapporte quantité de spécimens botaniques et minéralogiques qui seront intégrés aux collections du Musée de l'Instruction publique. Entre 1881 et 1887, le naturaliste français Henri de Puyjalon explore également ces régions pour le compte du gouvernement. Les rapports de Puyjalon, adressés au Commissaire des Terres de la Couronne, sont publiés à plusieurs reprises dans les Documents de la session ${ }^{87}$. Le naturaliste est l'un de ceux qui contribuent le plus à entretenir le mythe d'une Californie du Nord, mais beaucoup d'autres «explorateurs» y concourent. Retenons l'arpenteur Henry O'Sullivan, qui propose la colonisation de l'Abitibi et l'ouverture d'un chemin de fer vers la baie de James, l'ingénieur minier Joseph Obalski, qui explore la région de Chibougamau, H.-É. Faucher de SaintMaurice, membre de l'Assemblée et littérateur, qui vante également les richesses inépuisables du golfe et de la Côte Nord, le journaliste A.-N. Montpetit, qui étudie les rivières à saumon du Québec à la fin du siècle, etc. Sans oublier, au milieu de toute cette agitation, la Commission géologique qui poursuit méthodiquement son travail: en 1885, par exemple, John Macoun accompagne l'expédition de John Bignell et Albert P. Low au lac Mistassini. À titre de «géologue-adjoint» de la Commission, l'abbé Laflamme fait de nombreux relevés géologiques dans la région comprise entre le Saguenay et la rivière Saint-Maurice entre 1883 et 1901.

Ces expéditions permettent d'enrichir les collections du Musée de l'Instruction publique. Celui-ci se spécialise d'ailleurs dans les collections de la flore et de la faune nationales: «Je n'ai jamais perdu de vue, déclare Saint-Cyr en 1890, que ce devait être avant tout une oeuvre nationale, c'est-à-dire composée d'éléments, de matériaux indigènes.» ${ }^{88} \mathrm{C}$ 'est cet objectif également qui guide le conservateur dans l'acquisition de collections privées, même si les spécimens étrangers sont tout aussi accessibles ${ }^{89}$. En fait, les explorations et leur prolongement, le musée, servent à alimenter dans l'esprit des politiciens et d'une poignée de spéculateurs le rêve d'une prise de possession des territoires du Moyen Nord. Prenant la parole devant la Société de géographie, Benjamin Sulte donne le ton:

Ce que l'on veut, c'est occuper le territoire en utilisant la science moderne... Animés de ce sentiment, les Européens ont exécuté

\footnotetext{
87 Voir, par exemple, les Documents de 1885 (48 Victoria).

88 «Rapport du Surintendant de l'Instruction publique», Documents de la session, 1890 (54 $\underset{89}{\text { Victoria) }}$

${ }^{89}$ Le musée acquiert les collections entomologiques de Provancher à sa mort, en 1892 . I recueillera également celles du Révérend T. W. Fyles, entomologiste de Québec.
} 
des merveilles depuis dix ou douze années; ils ont découvert, étudié et fait connaître le quart de l'Afrique. Il ne leur reste qu'à envoyer des marchandises. Cela ne saurait tarder. Les nègres vont être civilisés par le calicot et les miroirs de poche.

Ce qui manque aux pays d'Europe, nous l'avons à nos portes: le territoire, l'espace, les ressources d'un sol fertile. Reste à en faire la géographie à tous les points de vue que cette science comporte. ${ }^{90}$

Dans le même esprit, Faucher de Saint-Maurice déclare à l'Assemblée:

Pourquoi la province de Québec n'étendrait-elle pas sa surveillance sur la partie du Labrador canadien qui lui appartient? Que faut-il pour cela? [...] Que la province de Québec songe aux richesses ignorées qui dorment sur cette partie de son territoire, et qu'elle encourage l'exploration. ${ }^{91}$

Instrument d'une appropriation symbolique du territoire «national», le Musée de l'Instruction publique a un autre rôle à jouer à travers ses collections: alimenter la participation du Québec aux expositions universelles. On sait le rôle que celles-ci jouent au XIXe siècle dans la célébration du progrès technique et du colonialisme. Emporté par le mouvement, comme d'ailleurs le reste du Canada et les Dominions de l'Empire, le Québec prend part à ces événements en y exposant non seulement les produits de son industrie ou de son agriculture, mais aussi ses richesses naturelles. La Centennial Exhibition de Philadelphie, en 1876, est la première exposition universelle à laquelle le gouvernement du Québec peut participer officiellement. Favorablement impressionné par les modestes succès que la province y remporte, Ouimet, qui a visité Philadelphie, décide de prendre en main la participation du Québec aux expositions suivantes. L'Exposition provinciale de Québec, en 1877, sert de répétition générale à l'Exposition universelle qui doit avoir lieu à Paris l'année suivante et où les maisons d'éducation de la province se présenteront en force ${ }^{92}$. À Paris, une brassée de prix et de médailles récompense les efforts du surintendant de l'Instruction publique et des exposants. Aussi ne s'étonne-t-on pas de voir Ouimet et ses successeurs, «imbus de cette grande vérité qu'il faut mériter les sympathies des hommes de science pour acquérir ensuite la confiance des hommes d'affaires» ${ }^{93}$, orchestrer la participation du Québec à l'Exposition coloniale de Londres en 1886, à la Columbian Exhibition de Chicago en 1893, à l'Exposition universelle de Paris en 1900, sans oublier une foule d'expositions secondaires. Tout comme le Musée de la Commission géologique, le Musée de l'Instruction publique servira de réserve

90 Cité par Morissonneau, 63-64.

91 Débats de la Législature (1882).

92 L.-P. Audet, «Le Québec à l'Exposition internationale de Paris en 1878», Cahiers des Dix, 32 (1967): 125-155.

93 Discours du Premier ministre Chapleau, Débats de la Législature (1882): 1532. 
aux trésors d'histoire naturelle exposés régulièrement au pays et à l'étranger.

Le Musée de l'Instruction publique ne sortira pas de ce rôle même si, à compter de 1900, la vogue des explorations et des expositions s'essouffle un peu. Sentant la chose, les successeurs de Saint-Cyr, l'abbé Huard en particulier, feront de modestes efforts pour donner un peu de popularité au Musée. Par exemple, on acquiert quelques spécimens plus spectaculaires, tel un ours blanc, acheté par Boucher de la Bruère, le successeur de Ouimet, à Paris en 1900, ou une famille complète de boeufs musqués. Huard, qui a repris le Naturaliste canadien, utilise également le journal pour faire un peu de publicité au musée provincial. Malheureusement, ces mesures ne suffisent pas à en faire un véritable musée populaire. Peu accessible, il n'accueille qu'une centaine de visiteurs par mois entre 1913 et $1918^{94}$. Plus de la moitié sont des touristes américains et canadiens; à peine $10 \%$ sont des habitants de la ville de Québec et de ses alentours. Le musée disparaît peu à peu dans l'indifférence générale. L'herbier de Saint-Cyr tombe en poussière ${ }^{95}$. Quant aux autres collections, elles sont progressivement dispersées au hasard des déménagements qui précèdent l'inauguration du Musée du Québec sur les plaines d'Abraham ${ }^{96}$.

Le Québec n'a donc jamais eu de véritable musée public d'histoire naturelle. Outre la pauvreté relative de l'État provincial et sa timidité dans le domaine de l'éducation en général, la raison en est peut-être l'absence au Canada français d'une tradition philanthropique comparable à celle qui a contribué au succès de beaucoup de musées américains. Là où les pouvoirs publics créent des musées d'histoire naturelle au XIXe siècle, ils ne font souvent que prendre le relais d'initiatives privées et de fondations philanthropiques.

\section{CONCLUSION}

Marquée par les vagues successives des musées de sociétés, des musées d'enseignement et des musées publics, l'histoire des musées et cabinets scientifiques du Québec ne se distingue pas substantiellement de celle des institutions étrangères. Liées à la popularité de l'histoire naturelle, le goût des collections et la fréquentation des musées de sociétés savantes - cette «conspicuous consumption» dont parlait Veblein - font partie du style de vie des classes aisées. Loisir distingué, l'histoire naturelle fait également partie de la formation que s'efforcent de donner aux jeunes gens les collèges. Ceux-ci pratiquent d'ail-

94 ANQ: Fonds du Secrétariat de la province; E0006/553-554-555; Registre des visiteurs au Musée de l'Instruction publique, 1908-1918.

95 Marie-Victorin n'en retrouve presque rien en 1923. APSC: Fonds Huard, 271-274; lettre de Marie-Victorin à Huard, 4 décembre 1923.

96 J. R. Barron, «Provancher's Collections of Insects, Particularly Those of Hymenoptera», Naturaliste canadien, 102,4 (1975): 387-591. 
leurs une politique de prestige à travers leurs collections. La manie de l'accumulation et de la classification trouve même une résonance dans les rêveries géopolitiques à l'origine du Musée de l'Instruction publique.

Cette popularité de l'histoire naturelle s'explique davantage par la mode et les mentalités que par une curiosité scientifique véritable ou par des objectifs de professionnalisation des sciences. Si le Québec compte beaucoup de naturalistes amateurs, en fait, il y a peu de véritables savants: tout au plus une dizaine, regroupés au sein de la Commission géologique, à l'Université Laval ou autour de Dawson à l'Université McGill, qui soient réellement engagés dans des recherches taxonomiques originales ${ }^{97}$. Peut-être cela explique-t-il le peu d'impact qu'a eu au Québec français la thèse de Darwin, qui remet pourtant en question le sens même de la taxonomie? Oeuvrant à la surface des choses, les amateurs poursuivent tout bonnement leur chasse aux spécimens.

À compter de 1900, on constate une profonde modification de l'histoire naturelle, aussi bien en tant que discipline scientifique que comme forme de loisir populaire. La discipline éclate littéralement, donnant naissance à une poignée de spécialités, telles la zoologie, l'ornithologie, etc. Plus important encore, les études sur le terrain - les «field-studies» - de spécimens vivants, évoluant dans leur habitat naturel, remplacent l'étude des espèces dans les musées. La vieille histoire naturelle, fondée sur la taxonomie et les collections, disparait, cédant la place à l'écologie. En tant que loisir populaire, l'histoire naturelle perd du terrain, phénomène dont les musées subissent le contrecoup. Les historiens se perdent en conjectures sur les raisons profondes de ce revirement ${ }^{98}$. Bien sûr, la botanique ou l'ornithologie conservent de nombreux fidèles après $1900^{99}$, mais l'histoire naturelle ne retrouvera jamais plus les légions d'amateurs qu'elle a connues au XIXe siècle. Les modes sont par nature changeantes, mais n'y aurait-il pas à ce déclin relatif de l'histoire naturelle des causes identifiables? Ce déclin fut-il un effet de la concurrence d'autres formes de loisirs, comme le tourisme ou le cinéma par exemple? Le caractère de plus en plus spécialisé et complexe des questions scientifiques finit-il par rebuter un public d'amateurs? En gagnant en popularité auprès des classes populaires, cette forme de loisir a-t-elle été victime de son propre succès, se trouvant de ce fait banalisée, «dévaluée» comme marque de distinction?

Quoi qu'il en soit, la désaffection du public à l'égard des musées d'histoire naturelle dans le monde fut à peine compensée par le succès

97 R. Duchesne, «Science et société coloniale: les naturalistes du Canada français et leurs correspondants scientifiques (1860-1900)», Scientia canadiensis, 5,2 (1981): 99-139.

98 Sheets-Pyenson, Cathedrals of Science, 93-96; Barber, 286-295.

99 Marianne G. Ainley, From Natural History to Avian Biology, Canadian Ornithology, 1860-1950, thèse de doctorat, Université McGill, 1985. 
de la nouvelle muséologie qui, dans le premier quart du XXe siècle, entreprit de présenter les spécimens dans une perspective «écologique» et plus dynamique. Au Québec comme ailleurs, une page était tournée dans l'histoire de la culture et les musées scientifiques étaient entrés dans une phase moins brillante de leur histoire. 